\title{
Microbunching-instability-induced sidebands in a seeded free-electron laser
}

\author{
Zhen Zhang, ${ }^{1,2}$ Ryan Lindberg, ${ }^{3}$ William M. Fawley, ${ }^{1}$ Zhirong Huang, ${ }^{1}$ Jacek Krzywinski, ${ }^{1}$ \\ Alberto Lutman, ${ }^{1}$ Gabriel Marcus, ${ }^{1}$ and Agostino Marinelli ${ }^{1}$ \\ ${ }^{1}$ SLAC National Accelerator Laboratory, Menlo Park, California 94025, USA \\ ${ }^{2}$ Department of Engineering Physics, Tsinghua University, Beijing 100084, China \\ ${ }^{3}$ Advanced Photon Source, Argonne National Laboratory, Argonne, Illinois 60439, USA
}

(Received 18 February 2016; published 2 May 2016)

\begin{abstract}
Measurements of the multishot-averaged, soft x-ray, self-seeding spectrum at the LCLS free-electron laser often have a pedestal-like distribution around the seeded wavelength, which limits the spectral purity and can negatively affect some user applications not employing a post-undulator monochromator. In this paper, we study the origins of such pedestals, focusing on longitudinal phase space modulations produced by the microbunching instability upstream of the free-electron laser (FEL) undulator. We show from theory and numerical simulation that both energy and density modulations can induce sidebands in a high-gain, seeded FEL whose fractional strength typically grows as the square of the undulator length. The results place a tight constraint on the longitudinal phase space uniformity of the electron beam for a seeded FEL, possibly requiring the amplitude of long-wavelength modulations to be much smaller than the typical incoherent energy spread if the output sideband power is to remain only a couple percent or less of the amplified seed power.
\end{abstract}

DOI: 10.1103/PhysRevAccelBeams.19.050701

\section{INTRODUCTION}

Many efforts have been devoted to improve the longitudinal coherence and spectral purity of x-ray free-electron lasers (FELs) since the unequivocal success of facilities such as FLASH [1], LCLS [2], and SACLA [3] that employ high-gain, self-amplified spontaneous emission (SASE). While the normalized output bandwidths of SASE FELs typically exceed values of $10^{-3}$ or larger, one can decrease the bandwidth and increase the longitudinal coherence by initiating the FEL process with a coherent seed [4-8], or by imprinting the electron beam with a coherent density modulation (bunching) at the wavelength of interest [9-16]. Under ideal circumstances (i.e., a high-quality external seed of sufficient power, essentially uniform longitudinal electron beam properties, etc.), one can obtain completely coherent, high-power x-ray pulses whose bandwidths approach the Fourier transform limit. As an example of high spectral purity in a seeded FEL operating in the extreme ultraviolet wavelength range, Ref. [17] has reported a longitudinal coherence result very close to the Fourier transform limit.

However, imperfections of the electron beam and/or of the seed will reduce the quality of the seeded FEL output [18-21]. Measurements of the multishot-averaged selfseeded, soft x-ray radiation spectrum at the LCLS [7]

Published by the American Physical Society under the terms of the Creative Commons Attribution 3.0 License. Further distribution of this work must maintain attribution to the author(s) and the published article's title, journal citation, and DOI. often show a pedestal-like distribution around the seeded frequency. In the absence of a post-undulator monochromator, this contamination limits the spectral purity and may degrade certain user applications. Further studies have ruled out the possibility that the pedestals originate from spectrometer noise or the monochromator optics. There are strong indications that modulations of the electron beam's longitudinal phase space prior to the undulator, mostly induced by longitudinal space charge forces within the long-distance acceleration and drift sections [22,23] and directly observed at the LCLS recently [24], could be one of the main sources for these spectral pedestals. Similar pedestal-like spectra have also been observed in the hard $\mathrm{x}$-ray self-seeding experiments at LCLS [6]. In this case, the users are less sensitive to such contamination because a very efficient, crystal-based monochromator in the hard $\mathrm{x}$-ray user beam line filters out the pedestal. We also note that a recent experiment carried out at the FERMI facility has intentionally seeded microbunching in the low energy, laser heater region of the linac in order to generate sidebands that lead to multicolor, extreme-ultraviolet FEL pulses [25].

In this paper we show that both energy and density modulations on the electron beam at the undulator entrance can induce sidebands in a high-gain, seeded FEL amplifier configuration. Both theory and simulations are used to analyze the sideband content and show that its strength relative to the desired, amplified seeded signal grows quadratically with undulator length. Our results place a tight constraint on the needed longitudinal phase space uniformity in a seeded FEL to generate a very high quality spectral output. 


\section{THEORETICAL ANALYSIS}

To understand the basic physics of the spectral pedestal formation, we consider a two-frequency system: the seed and the sideband. The FEL is seeded by a monochromatic radiation whose frequency is at or near the natural FEL resonant frequency $\omega_{1}$ and the electron beam initially has a longitudinal long-wavelength modulation at frequency $\omega_{s}$. We describe the longitudinal phase space of the electron beam with the electron ponderomotive phase $\theta \equiv\left(k_{1}+k_{u}\right) z-\omega_{1} t$ and normalized energy deviation from resonance $\eta \equiv\left(\gamma-\gamma_{0}\right) / \gamma_{0}$, where $k_{1}=\omega_{1} / c$ and $k_{u}$ are the wave numbers of the seed radiation and undulator, respectively. We find the following dimensionless variables to be useful in the analysis:

$$
\begin{gathered}
\hat{z} \equiv 2 k_{u} \rho z, \\
\hat{\eta} \equiv \frac{\eta}{\rho}, \\
a_{\nu} \equiv \frac{e K[J J]}{8 \gamma_{0}^{2} m c^{2} k_{u} \rho^{2}} E_{\nu},
\end{gathered}
$$

where the normalized frequency $\nu=1+\Delta \nu \equiv \omega / \omega_{1}$ and $\nu=1$ corresponds to the resonant frequency. Here $K$ is the normalized field strength of the undulator, $\rho$ is the FEL Pierce parameter [26] and $[J J]$ is the Bessel function coupling factor. With these dimensionless variables, the pendulum equations of the two-frequency system can be written as

$$
\begin{gathered}
\frac{d \theta}{d \hat{z}}=\hat{\eta} \\
\frac{d \hat{\eta}}{d \hat{z}}=a_{1} e^{i \theta}+a_{s} e^{i \nu \theta}+\text { c.c. } \\
\frac{d a_{1}}{d \hat{z}}=-b_{1} \\
\frac{d a_{s}}{d \hat{z}}+i \frac{\Delta \nu}{2 \rho} a_{s}=-b_{s}
\end{gathered}
$$

with the bunching parameters at the seed and the sideband frequency given by

$$
\begin{aligned}
& b_{1} \equiv\left\langle e^{-i \theta}\right\rangle, \\
& b_{s} \equiv\left\langle e^{-i \nu \theta}\right\rangle .
\end{aligned}
$$

We use subscripts " 1 " and "s" to denote variables corresponding to the seed and the sideband, respectively. We also introduce the collective momentum variables as

$$
p_{1} \equiv\left\langle\hat{\eta} e^{-i \theta}\right\rangle
$$

$$
p_{s} \equiv\left\langle\hat{\eta} e^{-i \nu \theta}\right\rangle .
$$

\section{A. Initial energy modulation}

Let us first consider an initial beam energy modulation $A(\zeta)=A_{0} \cos \left(k_{s} \zeta\right)=\frac{\Delta \gamma}{\gamma} \cos \left(k_{s} \zeta\right)$ where $\zeta$ measures the distance from the electron beam head. Using the scaled energy variable, we have

$$
\hat{\eta}_{0}=\frac{\hat{A}}{2} e^{i \Delta \nu \theta}+\text { complex conjugate, }
$$

where the normalized modulation amplitude $\hat{A}=A_{0} / \rho$ and $k_{s}=\omega_{s} / c=\omega_{1} \Delta \nu / c$ is the wave number of the modulation. We will study small amplitude modulations with $\hat{A}<1$.

With the assumptions that $\left|a_{s}\right| \ll\left|a_{1}\right|,\left|b_{s}\right| \ll\left|b_{1}\right|$ and $\left|p_{s}\right| \ll\left|p_{1}\right|$, we can obtain the equations of the field amplitudes as

$$
\begin{gathered}
\frac{d^{3} a_{1}}{d \hat{z}^{3}} \approx i a_{1}, \\
\frac{d^{3} a_{s}}{d \hat{z}^{3}}+i \frac{\Delta \nu}{2 \rho} \frac{d^{2} a_{s}}{d \hat{z}^{2}} \approx i \nu a_{s}+\nu^{2} \hat{A} p_{1} .
\end{gathered}
$$

Equation (13) is the well-known FEL cubic equation $[26,27]$ with an inhomogenous solution

$$
a_{1}(\hat{z})=\sum_{l=1}^{3} D_{l} e^{-i \mu_{l} \hat{z}},
$$

where $D_{1,2,3}$ are the coefficients determined by the initial conditions and $\mu_{1,2,3}$ are the roots of the cubic equation

$$
\mu_{1}=1, \quad \mu_{2}=\frac{-1-\sqrt{3} i}{2}, \quad \mu_{3}=\frac{-1+\sqrt{3} i}{2} .
$$

If we consider the high-gain regime $\left(e^{\hat{z}} \gg 1\right), a_{1}(\hat{z})$ is dominated by the growing mode and we can take the simple asymptotic form of

$$
a_{1}(\hat{z}) \approx D_{3} e^{-i \mu_{3} \hat{z}} .
$$

For small energy detuning $|\Delta \nu|<\rho$ (i.e., the sideband frequency shift is small compared to the FEL gain bandwidth), the sideband equation (14) is simplified to

$$
\frac{d^{3} a_{s}}{d \hat{z}^{3}} \approx i a_{s}+i \hat{A} D_{3} \mu_{3}^{2} e^{-i \mu_{3} \hat{z}}
$$

The solution of Eq. (18) in the high-gain regime for $a_{s}(0)=0$ is 


$$
a_{s}(\hat{z})=-\frac{i \hat{A} D_{3}}{3} \hat{z} e^{-i \mu_{3} \hat{z}}=-\frac{i \hat{A}}{3} \hat{z} a_{1} .
$$

Consequently, the power ratio between the sideband and the amplified seed radiation along the undulator is

$$
\frac{P_{s}(\hat{z})}{P_{1}(\hat{z})}=\frac{\hat{A}^{2}}{9} \hat{z}^{2}=\frac{4}{9} A_{0}^{2} k_{u}^{2} z^{2}
$$

Equation (20) applies to both the lower and upper sidebands for frequency shifts much less than the FEL gain bandwidth in the high-gain regime. If we take FEL power saturation to occur at $2 k_{u} \rho z=9$, Eq. (20) states that $A_{0}<\frac{1}{3} \rho$ is required in order for the sideband to not exceed the seed power at saturation. We will give a numerical estimation of this requirement in Sec. IV.

The lack of a $\Delta \nu$ dependence in Eq. (20) indicates that this single-frequency sideband analysis can be easily generalized to a broadband sideband spectrum driven by modulations whose scale lengths are all very much longer than the coherence length $l_{c} \approx \lambda_{1} / 4 \pi \rho$ by

$$
\frac{P_{s}(\hat{z})}{P_{1}(\hat{z})}=\frac{\hat{A}^{2}}{9} \hat{z}^{2}=\frac{\left(2 k_{u} \rho z\right)^{2}}{9} \int_{0}^{l_{b}} \frac{A^{2}(\zeta)}{\rho^{2}} \frac{d \zeta}{l_{b}} .
$$

Here we have presumed a flattop current profile with $A(\zeta)$ being the energy centroid modulation along the bunch coordinate $\zeta$ and $l_{b}$ is the electron beam bunch length.

To obtain a more general solution for arbitrary detuning, the cubic dispersion relation becomes

$$
\mu^{2}\left(\mu-\frac{\Delta \nu}{2 \rho}\right)=1,
$$

whose solutions we denote as $\bar{\mu}_{1}, \bar{\mu}_{2}, \bar{\mu}_{3}$. A useful approximation is

$$
\bar{\mu}_{i} \approx \mu_{i}+\frac{1}{3} \frac{\Delta \nu}{2 \rho}+\frac{1}{9 \mu_{i}}\left(\frac{\Delta \nu}{2 \rho}\right)^{2},
$$

where $\mu_{i}$ refers to the solutions given in Eq. (16).

The linear term in $\Delta \nu$ produces a simple eikonal phase shift while the quadratic term reduces the exponential growth rate and gives a $\hat{z}$-dependent, effective gain bandwidth $\Delta \nu_{\text {FWHM }}$ of $\approx 7.6 \rho \hat{z}^{-1 / 2}$, symmetric around $\Delta \nu=0$. For the particular situation where initially there is a broadband, noiselike excitation whose angular frequency cutoff significantly exceeds $\approx 10 \rho \omega_{1}$, Eq. (21) should be modified and will show a $z^{3 / 2}$ dependence due to the gain narrowing. In principle, both the observed sideband spectral width and the $P_{s}$ to $P_{1}$ ratio as a function of undulator length could be used to infer several spectral properties of the initial sideband modulation.

For initial conditions where all beam perturbation quantities are initially zero at $\hat{z}=0$ except the seed field $a_{1}(0)$, the growing sideband mode $a_{s}(\hat{z})$ is approximately given by

$$
\begin{aligned}
a_{s}(\hat{z}) \approx & i \hat{A}\left[\frac{e^{-i \mu_{3} \hat{z}} /(\Delta \nu / 2 \rho)}{\left(\mu_{3}-\mu_{2}\right)\left(\mu_{3}-\mu_{1}\right) \mu_{3}}\right. \\
& \left.-\frac{e^{-i \bar{\mu}_{3} \hat{z}} /(\Delta \nu / 2 \rho)}{\left(\bar{\mu}_{3}-\bar{\mu}_{2}\right)\left(\bar{\mu}_{3}-\bar{\mu}_{1}\right) \bar{\mu}_{3}}\right] a_{1}(0) .
\end{aligned}
$$

The power ratio of the sideband to the seed can be obtained by factoring out $e^{-i \bar{\mu}_{3} \hat{z}}$. Expanding the remaining terms to second order in $\Delta \nu / 2 \rho$ results in

$$
\begin{aligned}
\frac{P_{s}}{P_{1}} \approx & \frac{\hat{A}^{2}}{9}\left[1-\sqrt{3} \hat{z}+\hat{z}^{2}-\left(\frac{\Delta \nu}{2 \rho}\right) \frac{2-2 \sqrt{3} \hat{z}+\hat{z}^{2}}{6}\right. \\
& \left.+\left(\frac{\Delta \nu}{2 \rho}\right)^{2} \frac{12-16 \sqrt{3} \hat{z}+22 \hat{z}^{2}-4 \sqrt{3} \hat{z}^{3}-\hat{z}^{4}}{108}\right] .
\end{aligned}
$$

The above expression asymptotically approaches that of Eq. (20) for $e^{\hat{z}} \gg 1$ and $\Delta \nu \ll \rho$.

The above analysis is based on the standard FEL pendulum equations. The same conclusions can be obtained from applying a Maxwell-Vlasov approach to the coupled radiation/electron beam system. A detailed derivation for this alternate approach, valid both for arbitrary sideband frequency and FEL start-up parameters, has been worked out in Ref. [28].

In the Appendix, we present an alternate time-domain treatment that considers the spectral effects of a variation in the temporally local, complex gain length that might arise from a long wavelength modulation in one or more electron beam properties. The analysis shows that not only an energy modulation, but also similar, long-wavelength variations in quantities such as emittance, slice energy spread, and current all can induce sidebands in seeded FELs whose normalized strength grows quadratically with undulator length.

\section{B. Initial density modulation}

The above analysis can be applied in a straightforward manner to consider modulations in current. Let us introduce an initial, monochromatic bunching modulation at a discrete sideband frequency $\omega_{s}=\Delta \nu \omega_{1}$ :

$$
B=\left\langle e^{-i \Delta \nu \theta}\right\rangle
$$

The sideband field equation for a density modulation excitation is then

$$
\frac{d^{3} a_{s}}{d \hat{z}^{3}}+i \frac{\Delta \nu}{2 \rho} \frac{d^{2} a_{s}}{d \hat{z}^{2}} \approx i \nu a_{s}+i \nu a_{1} B
$$

Presuming a small detuning shift and using similar procedures as for the case of an energy modulation, we 
obtain a power ratio between the sideband and the seeding signal

$$
\frac{P_{s}(\hat{z})}{P_{1}(\hat{z})}=\frac{|B|^{2}}{9} \hat{z}^{2}=\frac{\left(2 k_{u} \rho z\right)^{2}}{9}|B|^{2},
$$

which also grows quadratically with both the initial density modulation amplitude and the undulator length.

Usually the longitudinal microbunching instability develops both energy and density modulations on the electron beam. The final sideband amplitudes are the superposition of both effects. Combining Eqs. (14) and (27), we find the equation for a sideband driven by both long wavelength energy and density modulations:

$$
\frac{d^{3} a_{s}}{d \hat{z}^{3}} \approx i a_{s}+\hat{A} p_{1}+i B a_{1}
$$

Then using the asymptotic limit of $p_{1} \approx i \mu_{3}^{2} a_{1}$, the sideband radiation field can be formulated as

$$
a_{s}(\hat{z}) \approx-\frac{i}{3}\left[\hat{A}+\left(-\frac{1}{2}+\frac{\sqrt{3}}{2} i\right) B\right] \hat{z} a_{1}(\hat{z}),
$$

and the power ratio to the seed is

$$
\frac{P_{s}(\hat{z})}{P_{1}(\hat{z})} \approx \frac{1}{9}\left|\hat{A}+\left(-\frac{1}{2}+\frac{\sqrt{3}}{2} i\right) B\right|^{2} \hat{z}^{2} .
$$

The final sideband power depends on the amplitude and phase difference between the two modulations, $\hat{A}$ and $B$. In a situation where an electron beam approaches the undulator region with a low frequency energy modulation (e.g., arising from longitudinal wake fields present in the final linac sections) and there also exists a moderately strong chromatic dispersion section (e.g., a dipole chicane used to allow the introduction of an external radiation seed), the combination will produce a density modulation with the same frequency. According to the definition of bunching factor in Eq. (26), the phase difference between the energy and induced density modulations for the upper sideband frequency $(\Delta \nu>0)$ is $90^{\circ}$, while for lower sideband frequency $(\Delta \nu<0)$ is $-90^{\circ}$. Examination of Eq. (30) shows that the net effect of the dispersion section is additional growth at the lower sideband and, in general, partial suppression of the upper sideband.

\section{SIMULATION RESULTS}

To verify the previous analytical considerations, we have numerically solved the time-dependent $1 \mathrm{D}$ FEL equations for a number of initial modulation conditions to study the growth of the sideband power. Here we adopt the parameters (see Table I) of a very recent, soft x-ray $(540 \mathrm{eV})$ selfseeding study [29] conducted at the LCLS [7]. The
TABLE I. Simulation parameters in 1D FEL code.

\begin{tabular}{lcc}
\hline \hline Parameter & Value & Unit \\
\hline Beam energy $E$ & 3.48 & $\mathrm{GeV}$ \\
Slice energy spread & 0 & $\mathrm{MeV}$ \\
Normalized emittance $\varepsilon_{N}$ & 0.9 & $\mu \mathrm{m}$ \\
Current $I$ & 1.4 & $\mathrm{kA}$ \\
Average $\beta$ & 30 & $\mathrm{~m}$ \\
Undulator period $\lambda_{u}$ & 3 & $\mathrm{~cm}$ \\
FEL parameter $\rho$ & $8.6 \times 10^{-4}$ & \\
Gain length $L_{G}$ & 1.6 & $\mathrm{~m}$ \\
Seeding wavelength $\lambda_{1}$ & 2.29 & $\mathrm{~nm}$ \\
Seeding power & 20 & $\mathrm{~kW}$ \\
\hline \hline
\end{tabular}

numerical simulations treat an idealized electron beam with a temporally uniform current distribution and zero slice energy spread. The initial seed power distribution is also temporally uniform. We choose sinusoidal energy modulations with wavelengths ranging from 2 to $10 \mu \mathrm{m}$; these lie within the range of the microbunching instability wavelengths observed in the experiment [24]. The initial modulation amplitudes range from 0.1 to $0.6 \mathrm{MeV}$, equivalent to $\hat{A}$ extending from 0.03 to 0.2 . The input seeding power of $20 \mathrm{~kW}$ leads to radiation power saturation at $\hat{z} \approx 9$.

The total FEL field amplitude $a$ and bunching factor $b_{1}$ at the seed frequency for different energy modulation amplitudes are plotted versus $\hat{z}$ in Fig. 1. The total radiation power, which is proportional to $a^{2}$, is almost completely independent of the energy modulation amplitude. However, the bunching factor $b_{1}$ is reduced near saturation for large energy modulation amplitude.

The FEL spectra along the undulator shown in Fig. 2 illustrate the growth of the sideband power. The spectra are normalized by the power of the amplified seed (main peak).

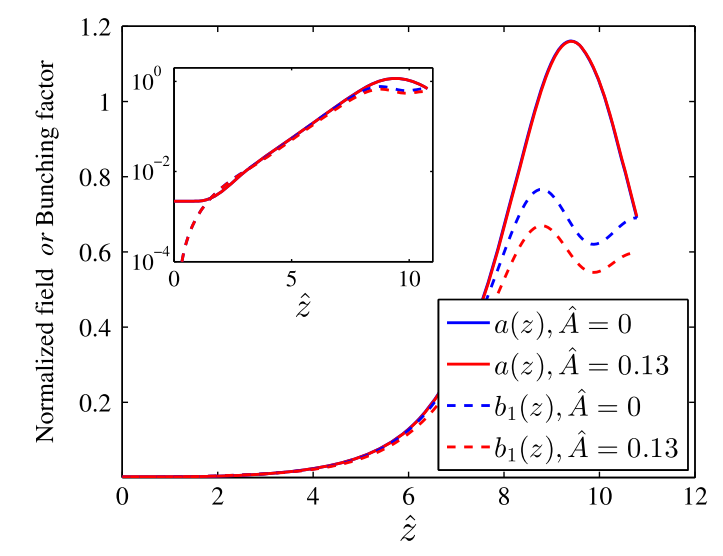

FIG. 1. The total normalized field amplitude $a$ and bunching factor $b_{1}$ along the undulator for various energy modulation amplitudes $\hat{A}$. The modulation wavelength is $8 \mu \mathrm{m}$, corresponding to $|\Delta \nu / 2 \rho| \approx 0.16$ The inset displays the same quantities on a logarithm scale. 


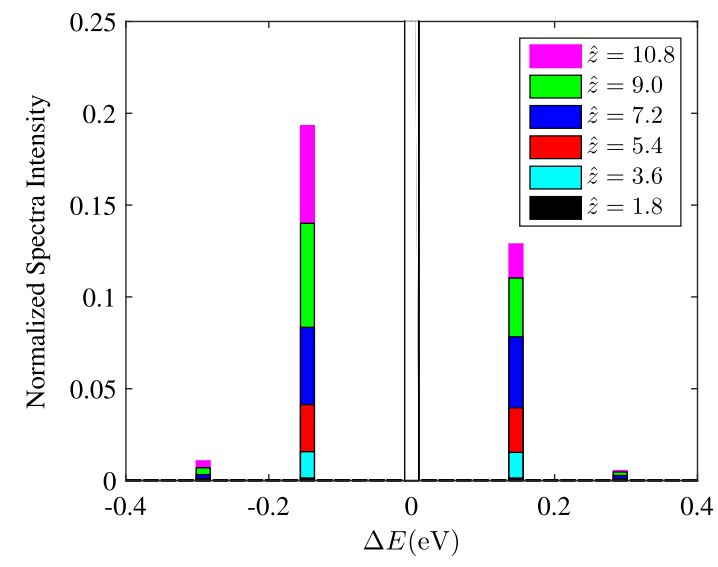

FIG. 2. Spectra along the undulator length to illustrate the growth of the sideband power. The spectra are normalized by the amplified seed intensity $a_{1}(\hat{z})$ and the spectral unit is photon energy $\left(\Delta E=\hbar \omega_{s}\right)$. The electron beam energy modulation wavelength is $8 \mu \mathrm{m}$ while the normalized modulation amplitude $\hat{A}=0.13$.

There are two sideband peaks near the seed and their normalized power increase along the undulator. The difference between the lower and upper sideband peaks is probably due to the smaller gain at the upper sideband [see Eq. (25)] and the density modulation developed as the power increases. Note that when the sideband strength becomes sufficiently large, second harmonics become evident with twice the energy offset but much smaller power. Here we only consider the first-order sideband as the higher harmonics are always very small in our cases of interest.

We also find that the energy offsets of the two sideband peaks to the seed peak are the same and proportional to the inverse of the modulation wavelength as predicted.

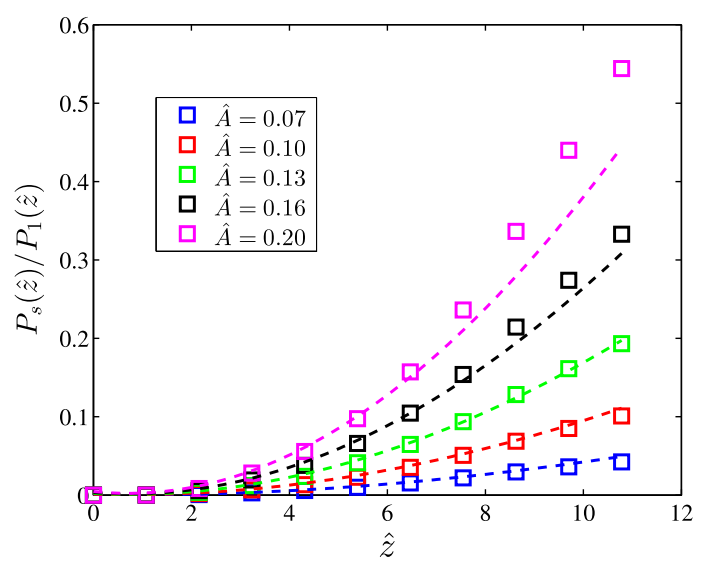

FIG. 3. The predicted power ratio of the lower sideband to the seed along the undulator for different energy modulation amplitudes in 1D simulations (squares). The energy modulation wavelength is $8 \mu \mathrm{m}$ and the corresponding $\Delta \nu / 2 \rho=-0.16$. All dashed lines are theory predictions from Eq. (25).
Simulation results show that the energy offset is independent of the modulation amplitude and undulator length.

The power ratios of the lower sideband to the seed peak along the undulator for different modulation amplitudes are shown in Fig. 3. The theoretical predictions of Eq. (25) are also given in the figure, which indicate that the sideband power ratio grows quadratically with $\hat{z}$ and $\hat{A}$ in the high-gain regime. The $1 \mathrm{D}$ simulations agree quite well with the theory predictions, especially when the power ratio is small.

Figure 4 presents the power ratio between the two sidebands for different detunings at the same energy modulation amplitude as predicted by 1D simulations. For $|\Delta \nu / 2 \rho| \gtrsim 0.5$, the sideband growth is sensitive to $\Delta \nu$ so we use the theory predictions obtained from Eq. (24) for comparison with the simulation results. For $\hat{z}<8$ (i.e., still well before saturation), the simulations are in very good agreement with the theory predictions for both sidebands. However, deviations begin to appear for larger $\hat{z}$ values. The power of the upper sideband increases more slowly and even begins to decrease as the fundamental enters the saturation regime. The deviations result in part from the reduction in electron beam energy and also from the density modulation that develops on the beam as the sideband power increases. As discussed in the previous section, the net effect of a sideband density modulation is to

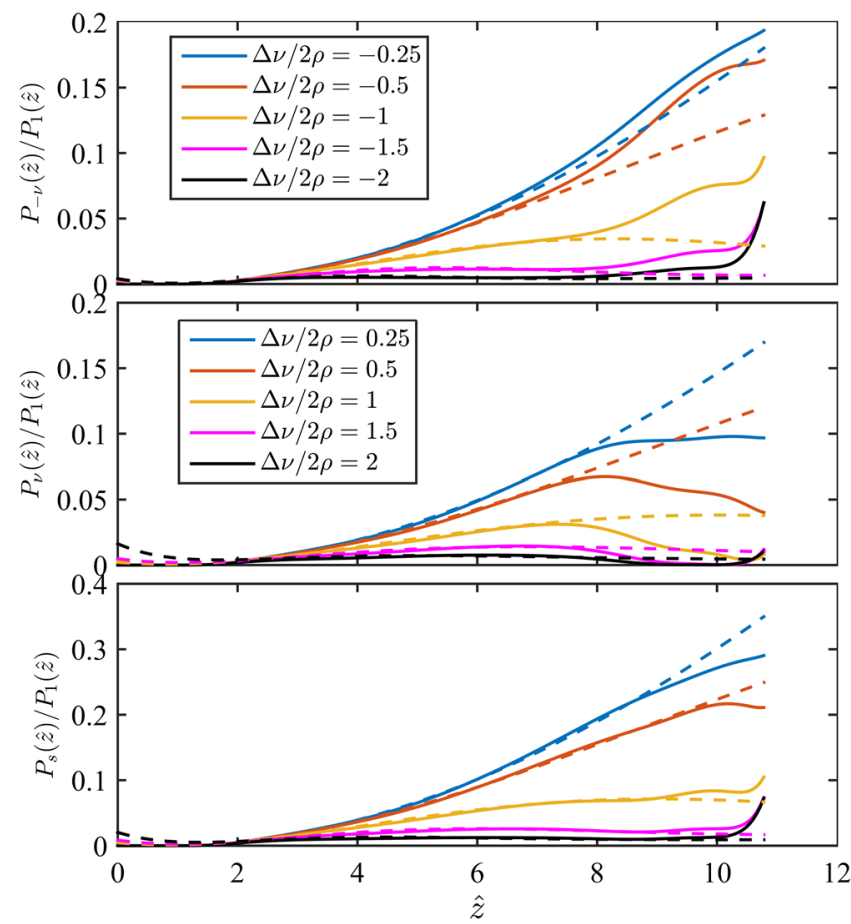

FIG. 4. The power ratio of the lower (top) and upper (middle) sidebands, and their sum (bottom) to the amplified seed along the undulator for different detunings (electron beam energy modulation wavelengths) according to 1D numerical simulations (solid lines). All dashed lines are the theory predictions of Eq. (24). The initial normalized energy modulation amplitude is $\hat{A}=0.13$. 


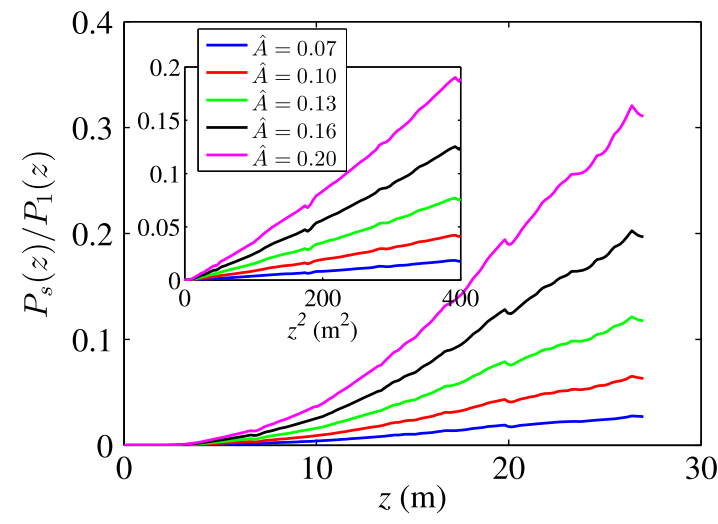

FIG. 5. The power ratio of the lower sideband to the amplified seed along the undulator for different modulation amplitude as predicted in 3D GENESIS simulations. The inset plots the power ratio versus the square of the undulator length.

give additional growth for the lower sideband and partial suppression for the upper sideband. The total power of the two sidebands does remain consistent with the analytic theory predictions (also shown in Fig. 4).

We also performed GENESIS [30] simulations to validate the $1 \mathrm{D}$ theory and simulations in the presence of $2 \mathrm{D}$ emittance, refractive and diffractive effects. We again adopt the electron beam and seed parameters of Table I, but now include the drift sections between adjacent LCLS undulator segments. The Rayleigh length of the input seed is about twice that of the FEL gain length, suggesting the 1D and 2D gain lengths will be quite similar. The energy offset of the sidebands is the same as found previously in the 1D simulations. Figure 5 displays the predicted sideband/seed power ratio versus undulator length; the figure's inset plots this ratio against the length squared, showing a clear $z^{2}$ dependence. Also note that the power ratio has a small drop at the beginning of each new undulator section. This occurs because the undulator break lengths are matched to the vacuum phase slippage occurring at the seed wavelength, thus leading to a net phase mismatch at the sideband radiation wavelengths.

The good agreement between the analytical predictions for sideband growth and those of the 1D and 2D numerical simulations suggests that the observed pedestal growth in the LCLS self-seeding experiments may well be due to long wavelength energy modulations. If so, the numerical limits corresponding to Eq. (20) place quite strict limits on the allowable modulation amplitude $A$ for those user applications (e.g., resonant inelastic x-ray scattering) that might require pedestal levels below a few percent of the amplified seed power. For example, obtaining a $1 \%$ or less pedestal fraction for a case with one-thousand-fold net seed amplification (i.e., $\hat{z} \approx 5.3$ when including launching losses) limits $A(\zeta)$ to $\approx 200 \mathrm{keV}$ amplitude for a $3.4-\mathrm{GeV}$ electron beam energy for an FEL parameter $\rho=10^{-3}$. Given that the typical operational value at the LCLS undulator entrance of the incoherent energy spread $\sigma_{E}$ is of order $500 \mathrm{keV}$ or greater, a $200 \mathrm{keV}$ limit on long wavelength energy modulations is not only extremely tight but also difficult to measure with normal electron beam spectrometer methods.

\section{CONCLUSIONS}

In the previous sections, we have investigated the effects of long wavelength residual energy and density modulations on the output of a seeded FEL. We have shown such modulations can generate sidebands of the seeded radiation if their frequency lies within the FEL gain bandwidth. A simple 1D theory is developed to estimate the sideband growth rate and agrees well with 2D numerical simulations that include emittance and diffraction effects. For sidebands whose frequencies lie very close to that of the seed (i.e., $\left.\Delta \omega \lesssim \rho \omega_{1} / 4\right)$, their power fraction in the exponential growth regime grows quadratically with the initial modulation amplitude and undulator length. Our analysis suggests that broadband modulations, such as might result from longitudinal microbunching instability growth, will result in a low amplitude "pedestal" in the output spectrum similar to that observed in self-seeding experiments at the LCLS [29]. We note that pedestals induced by longwavelength modulations will most likely appear in other seeding approaches that rely upon significant exponential growth, such as high-gain, harmonic generation schemes $[9,11]$ and also "advanced" SASE-configuration schemes that aim to narrow the output spectral bandwidth [31-33]. User applications that require a small ratio of pedestal power to amplified seed power may require the amplitude of long-wavelength modulations at the undulator entrance to be much smaller than the typical incoherent energy spread. Pedestal formation may also affect the efficiency of post-saturation undulator tapering as has been discussed previously in [28]; this particular subject likely merits further study given the strong interest in increasing the extraction efficiency of x-ray FELs.

\section{ACKNOWLEDGMENTS}

We thank D. Ratner, Y. Ding, T. Maxwell, C.-X. Tang and E. Allaria for useful discussions. This work was supported by U.S. Department of Energy Contracts No. DE-AC02-76SF00515 and No. DE-AC0206CH11357.

\section{APPENDIX: USING THE LOCAL COMPLEX EXPONENTIAL GROWTH RATE TO DETERMINE SIDEBAND GROWTH IN A HIGH-GAIN SEEDED FEL}

In contrast to Sec. II that used a discrete frequency approach to calculate the spectral broadening effects of long wavelength variations of electron beam parameters, here we calculate such effects using a time-domain 
analysis. This alternate approach in principle allows for situations where one or more other electron beam properties such as current, emittance, energy spread might also simultaneously vary with $\zeta$ on scale lengths much greater than the coherence length $l_{c}$. These variations too can lead to similarly slow temporal changes of the FEL Pierce parameter $\rho$, therefore perturbing both the instantaneous gain and gain bandwidth.

As before, we consider a situation where a relatively strong (as compared to the effective shot noise power), monochromatic radiation seed acts upon an intense, highly relativistic electron beam with nominal Lorentz factor $\gamma_{0}$ in an undulator whose magnetic strength $K_{R M S}$ is tuned for FEL resonance with the seed wavelength $\lambda_{1}$. We presume that the input seed power is much below the FEL saturation power and thus there will be exponential growth along the undulator. However, in contrast to the assumptions of Sec. II, for the case of an energy modulation we allow for both temporally local variations of $\gamma(\zeta)$ (e.g., a wakefield-induced energy chirp or a microbunching-induced sinusoidal energy variation) and shot-to-shot variability of the pulse-averaged $\gamma$ so that exact FEL resonance with the seed $\lambda_{1}$ may not apply neither locally in $\zeta$ nor, for a given shot, in a time-averaged sense. For the moment, we further assume that bulk diffraction, emittance and incoherent energy spread effects are sufficiently small that the 1D limit for the linearized FEL equations is an excellent approximation.

\section{Long wavelength energy modulation}

Turning first to the case of a temporally varying $\gamma$ within an individual pulse and presuming $\gamma_{0}$ is in proper FEL resonance with $\lambda_{1}$, the local detuning $\Delta \kappa(\zeta)$ is given by

$$
\begin{aligned}
\Delta \kappa(\zeta) & \equiv k_{u}-\frac{k_{1}}{2 \gamma^{2}(\zeta)}\left(1+K_{R M S}^{2}+\left\langle\gamma^{2} \beta_{\perp}^{2}\right\rangle\right) \\
& \approx+2 k_{u} \Delta \gamma(\zeta) / \gamma_{0}
\end{aligned}
$$

where the $\left\langle\gamma^{2} \beta_{\perp}^{2}\right\rangle$ term accounts for emittance effects. Let us make the standard eikonal approximation and express the total electric field as the product of a slowly varying term $\tilde{E}(z, t)$ times a rapidly varying quantity $\exp i\left(k_{1} z-\omega_{1} t\right)$ with $k_{1} \equiv \omega_{1} / c=2 \pi / \lambda_{1}$. For nonzero $\Delta \kappa$, the cubic eigenvalue equation for exponentially growing modes, $\tilde{E}(z, t) \sim \exp (-i \Gamma(\zeta) z)$, becomes

$$
\Gamma^{3}-2 \Delta \kappa \Gamma^{2}+\Delta \kappa^{2} \Gamma+\lambda^{3}=0
$$

where $\lambda \equiv 2 k_{u} \rho, \Delta \kappa$ and thus $\Gamma$ are all possibly slowly varying functions of $\zeta$.
We may rewrite this as

$$
\left(\Gamma-\frac{2}{3} \Delta \kappa\right)^{3}+\lambda^{3}-\Delta \kappa^{2} \Gamma+\frac{8}{27} \Delta \kappa^{3}=0 .
$$

If we presume $|\Delta \kappa| \ll 2 k_{u} \rho$, the last two terms may be neglected and one sees that the cubic roots $\Gamma_{1,2,3} \approx$ $2 k_{u} \rho(\zeta) \mu_{1,2,3}$ as given in Eq. (16). To lowest order in $\Delta \kappa$ and presuming a constant $\rho$, a variable energy modulation causes a shift in the $z$-derivative of the eikonal phase

$$
\frac{d}{d z} \Delta \phi(z, \zeta) \approx-2 \Delta \kappa(\zeta) / 3=-4 k_{u} \Delta \gamma(\zeta) / 3 \gamma_{0} .
$$

For a simple sinusoidal energy variation $\Delta \gamma(\zeta) / \gamma_{0}=$ $A \cos k_{s} \zeta$ with $k_{s} l_{c} \ll 1$, the eikonal phase at a fixed $z$ may be expanded as

$\exp [i \Delta \phi(z, \zeta)]=J_{0}(x)+2 \sum_{n=1}^{\infty} i^{n} J_{n}(x) \cos n k_{s} \zeta$,

with $x \equiv-4 A k_{u} z / 3$. The $n=1, J_{1}$ term corresponds to a sideband pair that is offset by wave number $\pm k_{s}$ from $k_{1}$; the terms for $n \geq 2$ are higher integral harmonics.

For $x$ small, the $J_{1}(x) \approx x / 2$ term is the dominant contributor giving

$$
\begin{aligned}
\exp [i \Delta \phi(z, \zeta)] \approx & 1+i x \times \frac{1}{2}\left(\exp \left[+i n k_{s} \zeta\right]\right. \\
& \left.+\exp \left[-i n k_{s} \zeta\right]\right)
\end{aligned}
$$

where the first and second terms in parentheses may be identified as the lower and upper sideband, respectively. Relative to the amplified seed component at wavelength $\lambda_{1}$, the sideband pair have identical normalized powers of

$$
\frac{P_{s}(\hat{z})}{P_{1}(\hat{z})}=\frac{4}{9} A^{2} k_{u}^{2} z^{2}
$$

in exact agreement with Eq. (20) of the frequency space analysis presented in Sec. II.

The temporally local angular frequency shift is simply $-d \phi(z, \zeta) / d \zeta$. On sees that for a situation where $\Delta \gamma$ has a linear chirp in time, the $\Delta \omega$ shift is constant over the pulse with a magnitude that linearly increases with $z$. A quadratic chirp similarly gives a linear frequency chirp, also increasing linearly with $z$. However, the effective dispersion parameter

$$
\left|R_{56}\right|=\frac{4}{3} \frac{k_{u}}{k_{1}} z
$$

is only two-thirds the value typically associated with an undulator of length $z$. A possible explanation for this discrepancy is that it is directly related to the same gain 
effect that lowers the effective group velocity of an exponentially growing mode such that its difference from the speed of light $c$ is two-thirds the ballistic slippage velocity $c \times k_{u} /\left(k_{u}+k_{1}\right)$.

For the case of LCLS with its warm linac configuration, there can be significant (when compared to $\rho$ ) shot-to-shot, fractional jitter in the mean electron energy at the undulator entrance. Unlike the situation for pure SASE configurations in which there is no constant, preselected wavelength associated with the input shot noise microbunching, a self-seeded scheme employing a monochromator does preselect such a constant wavelength $\lambda_{1}$. In this latter case, relatively strong energy jitter will on the average somewhat increase the gain length and thus require a slightly longer undulator length to reach a given average output power level. This effect is in addition to the well-known one associated with the statistical radiation power fluctuations immediately following narrow-band monochromatization of the SASE spectrum. In the case of sideband growth of the type studied here, a mean energy deviation on a given shot will lead to sidebands to one side of $\lambda_{1}$ having relatively stronger growth than those on the other side. Since presumably the coarse-average electron beam energy is chosen operationally to be in exact FEL resonance with the undulator $K, \lambda_{u}$, and monochromator-selected $\lambda_{1}$, the statistical effects of energy jitter will somewhat increase width of the pedestal but, presuming the fractional jitter level remains below $2 \rho$, are unlikely to significantly increase the total sideband power. These issues will be discussed further in the experimental analysis to be given in a future paper [29].

\section{Long wavelength current modulation}

To lowest order, a long wavelength current modulation is just a simple scaling of the temporally local gain. For the case of a simple sinusoidal modulation

$$
I(\zeta)=I_{0}\left(1+B \cos k_{s} \zeta\right)
$$

the local gain $\rho(\zeta) \approx \rho_{0}\left(1+\frac{1}{3} B \cos k_{s} \zeta\right)$. Consequently, the asymptotic radiation electric field for large $\hat{z} \equiv 2 k_{u} \rho_{0} z$ but $B \hat{z} \lesssim 1$ becomes

$a(\hat{z}, \zeta) \approx a_{1}(\hat{z})\left[1-\frac{i B}{3}\left(-\frac{1}{2}+\frac{\sqrt{3}}{2} i\right) \hat{z} \cos k_{s} \zeta\right]$,

where $a_{1}(\hat{z})$ is given by Eq. (17). This result recovers that of Eq. (28) when allowance is made that the Eq. (A9) perturbation is purely real while in the analysis of Sec. II it was complex.
[1] W. Ackermann et al., Nat. Photonics 1, 336 (2007).

[2] P. Emma et al., Nat. Photonics 4, 641 (2010).

[3] T. Ishikawa et al., Nat. Photonics 6, 540 (2012).

[4] J. Feldhaus, E. Saldin, J. Schneider, E. Schneidmiller, and M. Yurkov, Opt. Commun. 140, 341 (1997).

[5] G. Geloni, V. Kocharyan, and E. Saldin, J. Mod. Opt. 58, 1391 (2011).

[6] J. Amann et al., Nat. Photonics 6, 693 (2012).

[7] D. Ratner et al., Phys. Rev. Lett. 114, 054801 (2015).

[8] G. Lambert et al., Nat. Phys. 4, 296 (2008).

[9] L. H. Yu, Phys. Rev. A 44, 5178 (1991).

[10] L.-H. Yu et al., Science 289, 932 (2000).

[11] G. Stupakov, Phys. Rev. Lett. 102, 074801 (2009).

[12] D. Xiang et al., Phys. Rev. Lett. 108, 024802 (2012).

[13] Z. T. Zhao et al., Nat. Photonics 6, 360 (2012).

[14] E. Allaria et al., Nat. Photonics 6, 699 (2012).

[15] E. Allaria et al., Nat. Photonics 7, 913 (2013).

[16] H. X. Deng and C. Feng, Phys. Rev. Lett. 111, 084801 (2013).

[17] G. D. Ninno et al., Nat. Commun. 6, 8075 (2015).

[18] W. Fawley and G. Penn, Sincrontrone Trieste Technical Note No. ST/F-TN-06/07, 2006.

[19] A. A. Lutman, G. Penco, P. Craievich, and J. Wu, J. Phys. A 42, 085405 (2009).

[20] B. Jia, Y. K. Wu, J. J. Bisognano, A. W. Chao, and J. Wu, Phys. Rev. ST Accel. Beams 13, 060701 (2010).

[21] A. Marinelli, C. Pellegrini, L. Giannessi, and S. Reiche, Phys. Rev. ST Accel. Beams 13, 070701 (2010).

[22] E. L. Saldin, E. A. Schneidmiller, and M. V. Yurkov, Nucl. Instrum. Methods Phys. Res., Sect. A 490, 1 (2002).

[23] Z. Huang, M. Borland, P. Emma, J. Wu, C. Limborg, G. Stupakov, and J. Welch, Phys. Rev. ST Accel. Beams 7, 074401 (2004).

[24] D. Ratner, C. Behrens, Y. Ding, Z. Huang, A. Marinelli, T. Maxwell, and F. Zhou, Phys. Rev. ST Accel. Beams 18, 030704 (2015).

[25] E. Roussel, E. Ferrari, E. Allaria, G. Penco, S. Di Mitri, M. Veronese, M. Danailov, D. Gauthier, and L. Giannessi, Phys. Rev. Lett. 115, 214801 (2015).

[26] R. Bonifacio, C. Pellegrini, and L. M. Narducci, Opt. Commun. 50, 373 (1984).

[27] N. M. Kroll and W. A. McMullin, Phys. Rev. A 17, 300 (1978).

[28] R. Lindberg, ANL Technical Note No. AOP-TN-2015-028, 2015.

[29] G. Marcus et al. (to be published).

[30] S. Reiche, Nucl. Instrum. Methods Phys. Res., Sect. A 429, 243 (1999).

[31] B. W. J. McNeil, N. R. Thompson, and D. J. Dunning, Phys. Rev. Lett. 110, 134802 (2013).

[32] J. Wu et al., X-ray spectra and peak power control with iSASE, in Proceedings of the 4th International Particle Accelerator Conference, IPAC-2013, Shanghai, China, 2013 (JACoW, Shanghai, China, 2013).

[33] D. Xiang, Y. Ding, Z. Huang, and H. Deng, Phys. Rev. ST Accel. Beams 16, 010703 (2013). 